\title{
Gut bacterial metabolites of indigestible polysaccharides in intestinal fermentation as mediators of public health
}

\author{
Hijova E \\ Institute of Experimental Medicine, Faculty of Medicine, Safarikiensis University, Kosice, Slovakia. \\ emilia.hijova@upjs.sk
}

\begin{abstract}
OBJECTIVES: The gut microbiome is regarded as an essential dynamic organ that functions in nourishment, epithelial development and innate immunity. One important benefit of the dietary polysaccharides to human health is due to its fermentability in gut. It is been known quite well that dietary fiber is able of impacting colon microbiota. Fermented products from these polysaccharides, especially short-chain fatty acids (SCFAs) are bioactive molecules with health benefits. It is proposed that the dietary polysaccharide-deriving SCFAs could be converted into glucose and /or directly signal intestinal receptors and therefore contribute the benefits via gut-brain neural circuits. In addition, fermented polysaccharides can facilitate the beneficial bacteria to generate bioactive molecules important for the normal maturation of the host immune system. Manipulation of the microbiota and metabolites from intestinal microbiota might be a promising new approach for the prevention or treatment diseases (Fig. 2, Ref. 30). Text in PDF www.elis.sk. KEY WORDS: gut microbiota, dietary fibers, short-chain fatty acids, health benefit.
\end{abstract}

\section{Introduction}

Chronic over nutrition by unhealthy food high in energy dense, high-fat, and high-sugar, and low in natural polysaccharides, typically indigestible polysaccharides is considered as the key health risk factor for development of various diseases, metabolic conditions, local and systemic inflammation (Knudsen et al, 2018; Zhang et al, 2018). Polysaccharides are widely distributed in nature as molecules composed of long chains of monosaccharide units bound together by glyosidic linkages. These indigestible polysaccharides are often called dietary fiber.

The diet consists of some foods that contain dietary fibers, which are nondigestible food components that resist digestion in the upper gastrointestinal tract and can be utilized as fermentation substrate by resident microbiota. Some of these dietary fibers can be utilized as a specific substrate to allow competitive inhibition of intestinal pathogenic microbiota to result in specific changes in the intestinal microbiome and/or the activity of the gastrointestinal microbiota to confer health benefit to the host. These dietary fibers are defined as prebiotics. As defined by current prebiotic definition, in order for a dietary fiber to be classified as a prebiotic, the dietary fiber must meet 3 primary criteria for its classification, 1) it must resist digestion in the upper gastrointestinal tract, 2) it must be fermented by resident large intestinal microbiota, and

Address for correspondence: E. Hijova, MVDr, PhD, Institute of Experimental Medicine, Faculty of Medicine, LF UPJS, SNP 1, SK-040 11 Kosice, Slovakia. Phone: +421.55 .2343463$

Acknowledgements: This work was supported by projects APVV-16-0176 and project with ITMS 26220120058.
3) it must selectively stimulate the growth and /or activity of the gastrointestinal microbiota, to confer health benefits to the host.

Unlike a prebiotic, fermentable dietary fiber may only meet the first two criteria, but not the third criterion, and dietary fibers that are not fermented to any extent, such as insoluble, cellulosic fibers, also do not meet the last two criteria and are certainly not considered candidates as prebiotics. As a consequence, all prebiotics are classified as dietary fibers, but not all fibers are considered prebiotics. Most of polysaccharides are considered resistant to digestion in our alimentary system including the resistant starch that is mainly fermented by the large intestinal microbiota. A category of nondigestible carbohydrates that represent attributes that appear to go beyond those of a prebiotic, the human milk oligosaccharides, will be also discussed in detail. Therefore, the focus of this review are polysaccharides extracted from various food resources, which mainly contribute their health beneficial functions via their intestinal fermentability.

\section{Role of gut microbiota in polysaccharides degradation and fermentation}

The human gastrointestinal tract is composed of the most heterogenous group of microorganisms that is rich in species, but unique as a fingerprint for each individual. This community of microorganisms is defined as the gut microbiota, and its collective genomes are known as the gut microbiome (Peterson et al, 2009). Microbiome can be regarded as a ubiquitous, symbiotic, and essential organ of the human body, responsible for functions that human cells are unable to carry out. A substantial part of this organ is located in the human gut, the natural habitant of a com- 


\section{7-812}

plex microbial community comprising species of archaea, bacteria, viruses, and eukaryotes. Most of these microbes are mutualistic symbionts promoting human health through their contributions to nutrient processing, colonization resistance, immune system development, and stimulation of a wide variety of other host functions. Gut microbial community development is an example of ecological succession, starting when the embryonic intestinal organ is developing in the uterus (Vallés et al, 2014).

The taxonomic classification of the intestinal microbiota according to the ordinary nomenclature (phylum-class-order-family-genus-species) has characterized the most common phyla Firmicutes, Bacteroidetes, Actinobacteria and Proteobacteria. Gut microbiota has an extensive impact on both normal human physiology and disease susceptibilities, including defense against pathogens, nutrient utilisation, and peripheral education of the immune system.

Microbiota function (Koropatkin et al, 2010; Brusaferro et al, 2018):

a) metabolite production - the fermentation of complex carbohydrates results in the production of short-chain fatty acids (SCFAs), which are involved in many cellular processes and metabolic pathways, in the enhancement of the gut barrier function and in the regulation of immune system and inflammatory responses,

b) metabolic organ - with enzymatic properties that enhance or supersede our own, such as the ability to degrade resistant dietary or host-derived glycans that transit the distal gut, regulation of the bile acid metabolism, induction/protection from metabolic endotoxaemia,

c) vitamin production - microbiota synthetize essential vitamins that humans cannot produce (e.g., vitamin B12, vitamin K), a dysregulation results in metabolic pathologies such as obesity and diabetes mellitus type 2,

d) influence on epithelial homeostasis - microbiota promote epithelial integrity by influencing the turnover of epithelial cells and modulating mucus properties,

e) development of the immune system - both intestinal mucosal defense and the systemic immune system are modulated by microbiota, resulting in a greater protection against infections and against inflammatory diseases,

f) influence on pathogen colonization - microbiota compete with pathogens for attachment sites and nutrients, and they produce antimicrobial substances.

The rational nutrition with many natural products as food containing a great number of polysaccharides, degradation of which produces a large number of oligosaccharides that are conducive to host health is very important. Polysaccharides can serve as prebiotics in our daily diet, as a unique carbon sources for specific intestinal bacteria during fermentation and can promote the growth of probiotics bacteria and intestinal biodiversity. Polysaccharides, which cannot be processed by gastric and intestinal enzymes are degraded via the carbohydrate active enzymes (CAZymes) derived from intestinal microbiota.

Two major phyla dominate the human bowel microbiome kingdom, including the Gram-negative Bacteroidetes, which can degrade a relatively wide range of polysaccharides and the Gram- positive Firmicutes, which tend to metabolize a series of selected polysaccharides. The hydrolysis of polysaccharides happens only when they are transported to the cell surface of the bacteria. Therefore, the glycoside hydrolase, polysaccharide lyases and carbohydrate esterases in these bacteria must contain signal sequences for exportation to the surface of the cell. The mechanisms of polysaccharide degradation in bacteria involves three main system that are Sus-like transport sytem, ABC-transport system and cellulosome-like scaffolded enzyme system. The starch utilization system (Sus) of the Bacteroidetes thetaiotaomicron was the first described by Anderson and Salyers (1989a, 1989b). Sus in Bacteroidetes thetaiotaomicron degrades starch into maltooligosaccharides via SusG ( $\alpha$-amylase and part of a large protein complex), and maltooligosaccharides are transported into periplasm by TBDT (TonB-dependent transposrter) SusC through SuD, SusE and SusF (lipoproteins) and are degraded into maltose and glucose that are important into the cytoplasm.

The enzymes in the Sus-like transport system are encoded by the polysaccharide utilization loci (PUL) of the genome, which are genetic clusters encoding essential proteins for a capture, degradation, and importation of specific polysaccharides. ABC (ATP-binding cassette) transport system is another polysaccharide degradation system, which is common in the Firmicutes and Bifidobacterium. $\mathrm{ABC}$ in Eubacterium rectale degrades starch into maltooligosaccharides through cell surface amylases. Maltooligosaccharides are recognized by two separate $\mathrm{ABC}$ transport solute-binding proteins and then carried into the cytoplasm. Cellulosome-like scaffolded enzyme system in the Ruminococcus champanellensis mainly targets cellulose and resistant starch, brings the cellulose and multi-enzyme complexes (cellulosomes) together on the cell surface via the dockerin-cohesion protein to degrade celluloses into monosaccharides.

During the saccharolytic fermentation process, the commensal gut microbiota derives most of its nutrition from the nondigestible dietary carbohydrates, which in turn, produce fermentation products, such as short-chain fatty acids (SCFAs) mainly acetate, propionate and butyrate and bacterial proteins, that express a cascade of metabolic functions related to host nutrition and health (Hijová and Chmelárová, 2007). The chemical composition and physicochemical properties of the dietary carbohydrates influence the amount and composition of SCFAs produced during fermentation. SCFAs can be easily absorbed and promote the intestinal epithelial cells barrier function, promote gut homeostasis and epithelial proliferation, regulate immune response, regulate certain gene expression and may promote the growth of certain intestinal bacteria, thus changing the composition of intestinal microbiota and affecting the host health.

The SCFAs produced by the gut microbiota from dietary nondigestible carbohydrates are found in hepatic, portal and peripheral blood. SCFA are transported from the lumen across the apical membrane of the colonocyte into the colonocyte and then through the basolateral membrane of the colonocyte into portal blood. The SCFAs are either utilized by the gut epithelial cells for energy (butyrate and to a less propionate) or are diffused into the portal vein from the intestinal lumen and are then taken up by 
various peripheral organs, where they act as substrates or signaling molecules with key G-protein-coupled receptors (Gprs), to influence host energy homeostasis.

Some other beneficial actions for fiber and SCFAs can be summarized as followed: 1) "competitive exclusion", whereby a highfiber diet expands commensal bacteria and limits pathogenic bacteria access to the gut epithelium; 2) SCFA - induced promotion of mucous production by gut epithelial cells; 3) SCFA - induced secretion of IgA by B cells; 4) SCFA - induced promotion of tissue repair and wound healing; 5) SCFA - induced promotion of Treg cell development in the gut in a process that presumably facilitates immunological tolerance; 6) SCFA (particularly acetate) - mediated enhancement of epithelial integrity in a process dependent on inflammasome activation and IL-18 production; and 7) antiinflammatory effects, particularly inhibition of NFKB (Fig. 1), (Thorburn et al, 2014; Tungland, 2018).

SCFAs interact with the G-protein-coupled receptors (Gpr, now called Free Fatty Acid receptors, FFARs), which are expressed in various tissues including the colonic epithelial cells (Gpr109A) and immune cells (Gpr41 and Gpr43). Stimulation of the Gpr41/ FFAR3 and Gpr43/FFAR2 receptors promote the enteroendocrine secretion of peptide YY (PYY), which inhibits gastric emptying and intestinal transit time, thereby suppressing appetite and by promoting glucagon-like peptide 1 (GLP-1), the latter with stimulatory effects on insulin secretion. These two gut hormones reduce gut motility, promote satiety, and suppress energy intake. Gpr109A ac- tivated by butyrate, activates the inflammation-associated pathway in colonic macrophages and dendritic cells, resulting in differentiation of regulatory T cells, IL-10 producing T cells and increase secretion of IL-18 in intestinal epithelial cells (Thangaraju et al, 2009; Bindels et al, 2013). SCFA-Gpr41 and Gpr43 interactions stimulate leptin production and affect inflammatory responses that are responsible for the development of obesity-related metabolic disturbances such as insulin resistance, lipogenesis, and increased triglyceride stores. Finally, different SCFAs can have different metabolic properties, SCFAs involve interorgan connection to mediate glucose and lipid metabolism through adenosine monophosphateactivated protein kinase (AMPK) activation in liver and muscle as is shown in Figure 2 (Tungland, 2018).

Butyrate is produced from carbohydrates via glycolysis from the combination of two molecules of acetyl-CoA to form acetoacetyl-CoA, followed by stepwise reduction to butyryl-CoA. There are two different pathways for the final step in butyrate formation from butyryl-CoA. In the first pathway, butyryl-CoA is phosphorylated to form butyryl-phosphate and subsequently transformed to butyrate via butyrate kinase. In the second pathway, the CoA moiety of butyryl-CoA is transformed to acetate via butyryl-CoA: acetate CoA-tranferase leading to the formation of butyrate and acetyl-CoA. Butyrate is produced by endogenous intestinal bacteria Faecalibacterium prausnitzii and Eubacterium rectale, Roseburia spp., Eubacterium hallii and Ruminococcus bromii in the human colon (Rios-Covián et al, 2016). Butyrate is mainly used by colono-

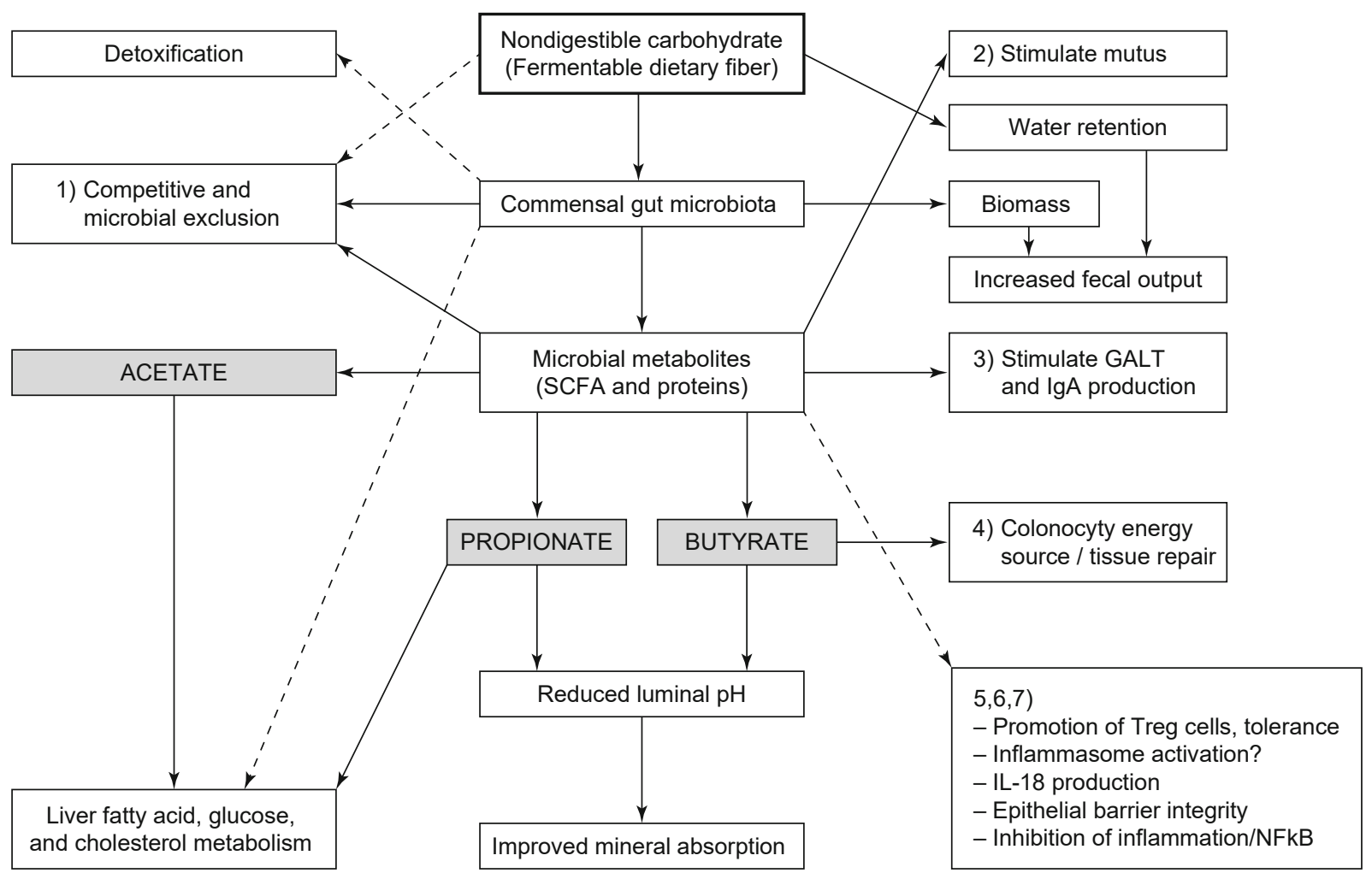

Fig. 1. Nondigestible carbohydrate (dietary fiber) utilization, SCFAs and gut homeostasis (Tungland, 2018). 
807-812

cytes as an energy source and is poorly detected in the circulation, exerts strong anti-infective and anti-inflammatory properties in the gut, its able to prevent increases in body weight without altering food intake or energy expenditure, improves insulin sensitivity, and decreases the respiratory exchange ratio (Henagan et al, 2015).

Propionate is dominated by relatively few bacterial genera, among which Akkermansia municiphila is the most important
(Rios-Covián et al, 2016). Propionate appears protective effect reducing the risk of cancer development and is associated with significant systemic metabolic effects, it is promptly absorbed and can be found in high concentrations in the circulation, increases the level of PYY, GLP-1, leptin, decreases serum cholesterol levels and liver lipogenesis, and induces satiety, thereby strongly contributing to weight control (Hosseini et al, 2011).

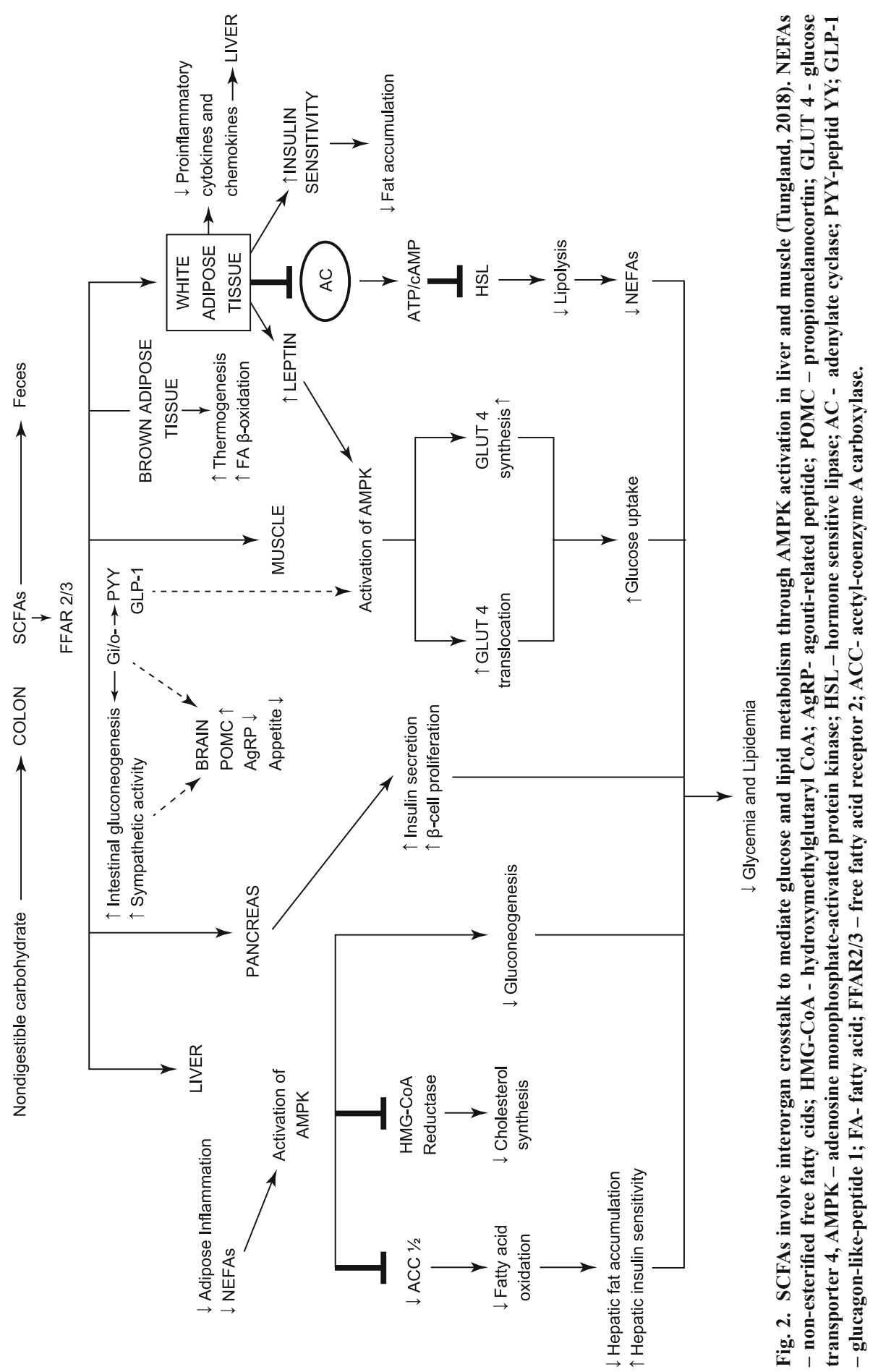

For years it was thought that an increased acetate production was a driver of metabolic syndrome, but it has been recently shown that rather than detrimental effects, the availability of a large amount of acetate can be beneficial, as it is associated with the reduction of appetite, a marked reduction of lipid accumulation in adipose tissue, protection against the accumulation of fat in the liver, and improved glucose tolerance (Frost et al, 2014; Everard et al, 2014).

Therefore, the natural polysaccharides benefit our health mainly by slowing gastric emptying, physically improving the bowel function, modulating the gut microbiome structure, working as substrate for microbial fermentation, and protecting the immune system. Imbalance, qualitative and quantitative modifications or decrease diversity of the gut microbiota referred as dysbiosis due to diet, antibiotic use, age and stress can be associated with the development of both intestinal and extra-intestinal disorders. Dysbiosis has been described in diseases including irritable bowel syndrome, inflammatory bowel disease, colorectal cancer, allergic diseases, arteriosclerotic diseases, and metabolic syndromes, most notably diabetes and obesity (Cani et al, 2008; Hijová et al, 2017 a,b).

Infant gut microbiota and human milk oligosaccharides as first prebiotic in our life

We must not forget that everything has a beginning and the establishment of our intestinal microbiota begins at birth and in some infants, colonization occurs 
in utero. Throughout the first year of life, microbial numbers and diversity increase, converting towards the microbiota of the adult. Mode of delivery, infant diet, and maternal or infant antibiotic treatment are the main early life exposures that influence microbial colonization and development in infancy. The development of the gut microbiome during infancy plays the crucial role in the maturation of immunologic and metabolic pathways. Compelling evidence supports the concept that shifts in the complex microbial system that occur early in life may confer an increased risk for developing obesity and other diseases later in life.

Human milk oligosaccharides (HMO) are highly abundant in human milk, their composition in human milk is determined by genetic factors and dependent on the mother's secretor (Se) and Lewis (Le) blood group characteristics. The different HMOs in four milk groups were characterized in preterm milk during the first month of lactation (Gabrielli et al, 2011). The health benefits of breastfeeding are partly explained by the abundant HMOs that serve as prebiotics substrates for specific commensal bacteria in the gut and help to shape the developing microbiome and immunomodulators of innate immune system in the infant gut. The HMOs may still serve as a prebiotic substrate for bifidogenic bacteria and support short chain fatty acid (SCFA) production and may also serve as receptor-mediated decoys for specific pathogenic bacteria. With the unique and complex carbohydrate structure, they resist gastrointestinal hydrolysis and digestion by pancreatic and brush-border enzymes and therefore are not absorbed in significant amounts. The prebiotic and immunomodulatory effects of HMOs may be particularly important for the population of very preterm infants to improve their intestinal maturation and protection. On the other hand, it is possible that the physiologically immature intestine in preterm infants hinders or changes the normal HMO-related improvements in the gut functions, microbiota composition and immune modulation. Supplemented human milk oligosaccharides (HMOs) may become more important for the gut protection in the preterm infants when the gut has reached a more mature phase. The bioactive components in human milk are highly important for the quenching of inflammatory processes after birth, by facilitating appropriate immune responses and antigenic memory. The HMO stimulation enhanced expression of genes involved in immune cell trafficking, proliferation and recruitment of immune cells to the mucosal surface. This could explain the clinical association between human milk consumption and reduced risk of preterm gut inflammation. The HMO particularly 2 '-fucosyllactose suppress CD14 expression in human intestinal epithelial cells, thereby attenuating lipopolysaccharide-induced inflammation. The inhibition of inflammation supports the role HMOs in innate immune system to protect the infant through the milk (Bering, 2018).

Infant gut microbiota could be a biomarker to identify the children, who are at risk of becoming overweight and obese later in childhood. Human studies conducted to date indicate that obesity may be associated with a reduced bacterial diversity and shifts of intestinal bacteria at the phylum level, however, discrepancies exist in the directionality and relevance of the Firmicutes to Bacteroidetes ratio in obesity. It is likely that obesity-related gut dysbiosis has its origins during infancy, as early as 3-6 months after birth, at a time, when first colonizers of gut microbiota lay the foundation for subsequent colonization by anaerobes from the Bacteroidetes phylum.

Epidemiological studies have published evidence on associations between infant gut microbiota and infant weight gain or later child overweight (Kalliomaki et al, 2008; Luoto et al, 2011; Vael et al, 2011; White et al, 2013; Scheepers et al, 2014). Human studies in adults point to the role for Bacteriodes spp., in particular Bacteroides fragilis (B. fragilis) are indeed key obesogenic microbes or simply an indicator of other aberrations in bacterial taxa that have a greater influence on weight development in early life. Obese children showed an elevated Firmicutes-to-Bacteroidetes ratio compared with lean children. High concentration of Lactobacillus spp. were observed in obese children. Additionally in obese children, Lactobacillus spp. were positively associated with plasma hs-CRP (Bervoets et al, 2013). Stanislawski et al (2018) concluded that gut microbiota composition at 2 years of age can be used as a predictor for obesity at age 12 . This finding suggests that gut microbiota composition may be the earliest warning sign for detecting obesity risk. Obesity is the pre-stage that leads to metabolic syndrome and the central feature of this condition is insulin resistance that greatly increases the risk for development of type 2 diabetes, cardiovascular diseases and other diseases.

\section{Conclusion}

As the major constituent of human milk, HMOs are probably significant contributors to general infant health during breast feeding. This could be due to their ability to stimulate the immune system and to provide substrates for development of a beneficial gut microbiota. The development of the microbiome from infancy to adulthood dependent on a range of factors, structural and functional assembly of the microbiome in early life to be involved in the pathobiology of later life diseases, provide a foundation for targeted mechanistic investigation into the consequences of microbial-immune crosstalk for long-term health. Many natural products as foods contain a great number of polysaccharides which are degraded and fermented with gut bacteria into short chain fatty acid that can effectively prevent or ameliorate symptoms of many chronic diseases. This is an emerging field, current leading metabolites that play protective roles can be supplemented and there might be many more.

\section{References}

1. Anderson KL, Salyers AA. Biochemical evidence that starch breakdown by Bacteroides thetaiotaomicron involves outer membrane starchbinding sites and periplasmic starch-degrading enzymes. J Bacteriol 1989a; 171: 3192-3198.

2. Anderson KL, Salyers AA. Genetic evidence that outer membrane binding of starch is ewquired for stacrch utilization by Bacteroides thetaiotaomicron. J Bacteriol 1989b; 171: 3199-3204.

3. Bering SB. Human milk oligosaccharides to prevent gut dysfunction and necrotizing enterocolitis in preterm neonates. Nutrients 2018; 10: 1461. 
807-812

4. Bervoets L, Hoorenbeeck K, Kortleven I, Noten C, Hens N, Goossens H, Desager KN, Vankerckoven V. Differences in gut microbiota composition between obese and lean children: a cross-sectional study. Gut Pathog 2013; 5: 1-10.

5. Bindels LB, Dewulf EM, Delzenne NM. GPR43/FFA2: Physiopathological relevance and therapeutic prospects. Trends Pharmacol Sci 2013; 34: 226-232.

6. Brusaferro A, Cozzali R, Orabona C, Biscarini A, Farinelli E, Cavalli E, Grohmann U, Principi N, Esposito S. Is it time to use probiotics to prevent or treat obesity? Nutrients 2018; 10: 1613.

7. Cani PD, Bibiloni R, Knauf C, Waget A, Neyrinck AM, Delzenne NM. Changes in gut microbiota control metabolic endotoxemia-indced inflammation in high-fat diet induced obesity and diabetes in mice. Diabetes 2008, 57: 1470-1481.

8. Everard A, Lazarevic V, Gaïa N, Johansson M, Ståhlman M, Backhed F, Delzenne NM, Schrenzel J, François P, Cani PD. Microbiome of prebiotic-treated mice revels novel targets involved in host response during obesity. ISME J 2014; 8: 2116-2130.

9. Frost G, Sleeth ML, Sahuri-Arisoylu M, Lizarbe B, Cerdan S, Brody L, Anastasovska J, Ghourab S, Hankir M, Zhang S, Carling D, Swann JK, Gibson G, Viardot A, Morrison D, Thomas EL, Bell JD. The shortchain fatty acid acetate reduces appetite via a central homeostatic mechanism. Nat Commun 2014; 5: 3611.

10. Gabrielli O, Zampini L, Galeazzi T, Padella L, Santoro L, Peila C, Giuliani F, Bertino E, Fabris C, Coppa GV. Preterm milk oligosaccharides during the first month of lactation. Pediatrics 2011; 128 : e1520-1531.

11. Henagan TM, Stefanska B, Fang Z, Navard AM, Ye J, Lenard NR, Devarshi PP. Sodium butyrate epigenetically modulates high-fat diet-induced skeletal muscle mitochondrial adaptation, obesity and insulin resistance through nucleosome positioning. Br J Pharmacol 2015; 172, 11: 2782-2798.

12. Hijová E, Chmelárová A. Short chain fatty acids and colonic health. Bratisl Lek Listy 2007; 108 (8): 354-358.

13. Hijová E, Kuzma J, Strojný L, Bomba A, Bertková I, Chmelárová A, Hertelyová Z, Benetinova V, Štofilová J, Ambro L. Ability of Lactobacillus plantarum LS/07 to modify intestinal enzymes activity in chronic diseases prevention. Acta Biochim Pol 2017 a; 69: 113-116.

14. Hijová E, Šoltésová A, Bertková I, Štofilová J, Strojný L, Bomba A. Improvement of colitis by Lactobacillus plantarum LS/07 and inulin. Acta Vet. Brno 2017 b; 86: 399-404.

15. Hosseini E, Grootaert C, Verstraete W, Van de Wiele T. Propionate as a health-promoting microbial metabolite in the human gut. Nutr Rev 2011; 69 (5): 245-258.

16. Kalliomaki M, Collado MC, Salminen S, Isolauri E. Early differences in fecal microbiota composition in children may predict overweight. Am J Clin Nutr 2008; 87: 534-538.
17. Koropatkin NM, Smith TJ. SusG: A unique cell-membrane-associated $\alpha$-amylase from a prominent human gut symbiont targets complex starch molecules. Structure 2010; 18: 200-2015.

18. Knudsen KEB, Lærke HN, Hedemann MS, Nielsen TS, Ingerslev AK, Nielsen DSG, Theil PK, Purup S, Hald S, Schioldan AG, Marco ML, Gregersen S, Hermansen K. Impact of diet-modulated butyrate production on intestinal barrier function and inflammation. Nutrients 2018; 10: 1499.

19. Luoto R, Kalliomaki M, Laitinen K, Delzenne NM, Cani PD, Salminen S, Isolauri E. Initial dietary and microbiological environments deviate in normal-weight compared to overweight children at 10 years of age. J Pediatr Gastroenterol Nutr 2011; 52: 90-95.

20. Peterson J, Garges S, Giovanni M, McInnes P, Wang L, Schloss JA, Bonazzi V, McEwen JE, Wetterstrand KA, Deal C. The NIH human microbiome project. Genome Res 2009; 19: 2317-2323.

21. Rios-Covián D, Ruas-Madiedo P, Margolles A, Gueimonde M, de Los Reyes-Gavilán CG, Salazar N. Intestinal short chain fatty acids and their link with diet and human health. Front Microbiol 2016; 7: 185.

22. Scheepers LE, Penders J, Mbakwa CA, Thijs C, Mommers M, Arts IC. The intestinal microbiota composition and weight development in children: The KOALA Birth Cohort Study. Int J Obes (Lond) 2014; 39: 16-25.

23. Stanislawski MA, Dabelea D, Wagner BD, Iszatt N, Dahi C, Sontag MK, Knight R, Lozupone CA, Eggesbe M. Gut microbiota in the first 2 years of life and the association with body mass index at age 12 in a Norwegian birth cohort. mBio. 2018; 9 (5): e01751-18.

24. Tungland B. Human Microbiota in Health and Disease: From Pathogenesis to Therapy 2018; Elsevier Inc., 649 p.

25. Thangaraju M, Cresci GA, Liu K, Ananth S, Gnanaprakasam JP, Browning DD, Mellinger JD, Smith SB, Digby GJ, Lambert NA, Prasad PD, Ganapathy V. Gpr109A is a G-protein-coupled receptor for the bacterial fermentation product butyrate and functions as a tumor suppressor in colon. Cancer Res 2009; 69 (7): 2826-2832.

26. Thorburn AN, Macia L, Mackay ChR. Diet, Metabolites, and "Western-Lifestyle“ Inflammatory Diseases. Immunity 2014; 40: 833-842.

27. Vael C, Verhulst SL, Nelen V, Goossens H, Desager KN. Intestinal microflora and body mass index during the first three years of life: An observational study. Gut Pathog 2011; 3: 8.

28. Vallés Y, Artacho A, Pascual-Garcia A, Ferrus ML, Gosalbes MJ, Abellán JJ, Francino MP. Microbial succession in the gut: Directional trends of taxonomic and functional change in a birth cohort of Spanish infants. PLoS Genet 2014; 10 (6): e.1004406.

29. White RA, Bjornholt JV, Baird DD, Midtvedt T, Harris JR, Pagano M, Hide W, Rudi K, Moen B, Iszatt N, Peddada SD, Eggesbo M. Novel development analyses identify longitudinal patterns of early gut microbiota that affect infant growth. PLoS Comput Biol 2013; 9: e1003042.

30. Zhang T, Yang Y, Liang Y, Jiao X, Zhao Ch. Beneficial effect of intestinal fermentation of natural polysaccharides. Nutrients 2018; 10: 1055.

Received May 7, 2019. Accepted June 10. 2019. 